\title{
Union hat kaum Gesundheitspolitik auf der Agenda
}

Fast 80 Seiten umfasst das Papier, das CDU und CSU Anfang Juli selbstbewusst als gemeinsames „Regierungsprogramm“ vorgelegt haben. Gesundheitspolitik gerät darin auf knappen drei Seiten eher als Pflichtübung, und die programmatischen Vorhaben lesen sich wie ein solides „Weiter so“, denn Gesundheit und Pflege seien hierzulande ja bereits auf hohem Niveau. "Sichern und ausbauen“ heißt die Devise, doch so richtig konkret wird die Union nicht. Ein Bekenntnis zur freiberuflichen Ärzteschaft und zur Selbstverwaltung ist abzulesen und die Ablehnung einer Bürgerversicherung. Eine Garantie wollen CDU und CSU allerdings geben: „gute ärztliche Versorgung im ländlichen Raum“. Ansonsten zeigt sich die Union eher zufrieden mit dem, was in den vergangenen Jahren erreicht wurde. Wörtlich heißt es: „Die vergangenen Regierungsjahre

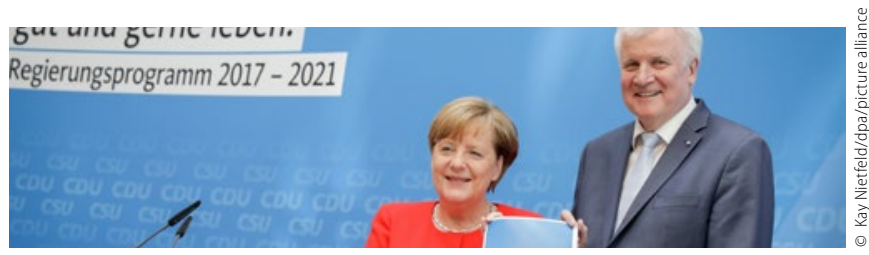

waren gute Jahre für Gesundheit und Pflege: Nach einer großen Zahl von Gesundheitsreformen in der Vergangenheit ist unser Gesundheitssystem nunmehr schon seit rund zehn Jahren stabil und leistungsfähig.“"

Mehr gesundheitspolitische Ideen der CDU/CSU und der anderen Parteien, die gute Chancen haben, dem nächsten Bundestag anzugehören, finden Sie im direkten Vergleich in den Wahlprüfsteinen des FVDZ ab Seite 18.

\section{Jahresbericht über zahnärztliche Patientenberatung}

\section{Prozent der Fälle gelöst oder abschließend bearbeitet}

Neben der direkten Beratung in der Praxis ist die kostenfreie zahnärztliche Patientenberatung der Kassenzahnärztlichen Vereinigungen und Landeszahnärztekammern für Patienten eine der bundesweit wichtigsten Anlaufstellen bei allen Fragen in Sachen Zahngesundheit. Das geht aus dem 1. Jahresbericht zur wissenschaftlichen Evaluierung des Beratungsangebotes hervor, den die Kassenzahnärztliche Bundesvereinigung (KZBV) und die Bundeszahnärztekammer (BZÄK) unter fachlicher Begleitung des Instituts der Deutschen Zahnärzte (IDZ) im Juni veröffentlichten.

Demnach konnte in knapp 90 Prozent der Fälle das Anliegen von Patienten gelöst oder an die zuständigen Kammern oder KZVen für die abschließende Bearbeitung vermittelt werden. Insgesamt gab es im Berichtsjahr 2016 fast 24.000 Beratungen.
„Unsere Beratung stärkt die Patientensouveränität und löst aktiv und häufig abschließend, statt bloßer Kummerkasten für unerwünschte Ereignisse zu sein“, sagte KZBV-Chef Dr. Wolfgang Eßer. Kein anderes Beratungsangebot schaffe es, Zahnarzt und Patient zeitnah zusammenzubringen und zwischen den Beteiligten lösungsorientiert zu vermitteln. Darin unterscheidet sich das Angebot aus Sicht der Zahnärzteschaft von Institutionen wie die „Unabhängige Patientenberatung Deutschland“.

Auch für den Bundesvorsitzenden des Freien Verbandes Deutscher Zahnärzte, Harald Schrader, sind die zahnärztlichen Beratungsstellen unverzichtbar: „Es ist viel besser, wenn die Beratung im Berufsstand bleibt, denn hier ist die Fachkompetenz und hier können wir individuell auf die Probleme der Patienten eingehen. Das können anonyme Callcenter nicht leisten." $\mathrm{mf}$

\section{Bundesgesundheitsministerium}

\section{Finanzreserven der Krankenkassen steigen}

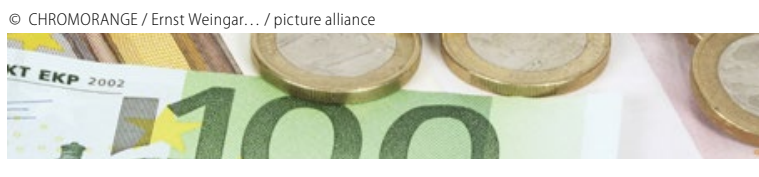

Frohe Kunde vor der Bundestagswahl aus dem Bundesgesundheitsministerium (BMG): Die anhaltend gute Konjunktur und die damit verbundene hohe Beschäftigung in Deutschland füllen die Kassen der gesetzlichen Krankenversicherung (GKV). Nach Angaben des BMG erzielten die gesetzlichen Kassen im 1. Quartal 2017 einen Überschuss von rund 612 Millionen Euro. Die Finanzreserven stiegen damit auf rund 16,7 Milliarden Euro. „Die gesetzliche Krankenversicherung steht auch weiterhin gut da", sagte Bundesgesundheitsminister Hermann Gröhe zu den Ergebnissen. „Das ist das Ergebnis einer sorgfältig abwägenden Gesundheitspolitik, die Einnahmen und Ausgaben gleichermaßen im Blick behält.“

Nach Angaben des Ministeriums liegen die Zuwachsraten bei den Einnahmen des Gesundheitsfonds (4,4 Prozent) angesichts der günstigen Entwicklung der Beitragseinnahmen deutlich über den Ausgabenanstieg der Kassen. Bei den Ausgaben für zahnärztliche Behandlung betrug der absolute Anstieg 3,2 Prozent (je Versichertem 2,0 Prozent), beim Zahnersatz 0,3 Prozent (je Versichertem 0,9 Prozent). Wie das BMG erläuterte, liegen bei den Krankenkassen für das erste Quartal in diesen Leistungsbereichen noch keine Abrechnungsdaten der Kassenärztlichen und Kassenzahnärztlichen Vereinigungen vor, und die ausgewiesenen Veränderungsraten haben lediglich Schätzcharakter. Bei der Betrachtung nach Kassenarten stellt das BMG fest, dass der Überschuss nicht bei allen Krankenkassen gleichermaßen ankommt. Während die Allgemeinen Ortskrankenkassen einen Überschuss von rund 361 Millionen Euro verzeichneten, lag dieser bei den Ersatzkassen nur bei 155 Millionen Euro und bei den Betriebskrankenkassen bei 27 Millionen Euro. Beitragsanhebungen für die Versicherten der GKV dürften angesichts dieser Zahlen allerdings weitgehend vom Tisch sein was sich im Wahljahr besonders gut macht. 